\title{
Improved All Cause and Cardiovascular Mortality and Morbidity by Empagliflozin: Too Good to Be True!
}

\author{
Udaya M. Kabadi ${ }^{1,2}$ \\ ${ }^{1}$ Broad Lawns Medical Center and Veterans Affairs Medical Center, Des Moines University, \\ Des Moines, USA \\ ${ }^{2}$ University of lowa, lowa City, USA \\ Email: ukabadi@gmail.com
}

Received 5 February 2016; accepted 17 April 2016; published 20 April 2016

Copyright (C) 2016 by author and Scientific Research Publishing Inc.

This work is licensed under the Creative Commons Attribution International License (CC BY). http://creativecommons.org/licenses/by/4.0/

c) (i) Open Access

It was interesting to read the recent article regarding the efficacy of Empagliflozin in reducing all cause and cardiovascular mortality [1]. Superficially, the report appears to be very promising. However, the methodology, the results and the conclusions deserve and need further scrutiny and validation for several reasons.

An important comparative datum between monotherapy with Empagliflozin and placebo is not included in the subgroup analysis. Additionally, no datum is provided between Empagliflozin and any other comparator such as sulfonylurea, metformin or any other antihyperglycemic agents used in management of type 2 diabetes. Subjects who received even a single dose of Empagliflozin and subjects who received it for a short duration as well as the dropouts were included in the final analysis. These subjects must be excluded from the analysis or should be included as a part of placebo group since they are not exposed to Empagliflozin at all or exposed for a short duration because of being dropouts, and therefore they are similar to subjects administered placebo. And if they are excluded from the analysis, the mortality in Empagliflozin group may rise and get closer to the placebo group. Alternatively, if these subjects are included in the placebo group, the mortality in placebo group may be lowered and get closer to Empagliflozin groups. Finally, 25\% dropout rate in Empagliflozin groups is apparently very high and may also have altered the results. Moreover, the comparisons between placebo group on one hand and the two individual groups of subjects treated with two different daily doses of Empagliflozin, $10 \mathrm{mg}$ and $30 \mathrm{mg}$ yielded no significant differences. Therefore, it is difficult to accept the finding of a statistically significant difference for cardiovascular outcomes between subjects administered placebo and the overall population of subjects treated with Empagliflozin including both dosage groups. Finally, it is ludicrous to conclude that even one prescription or a single dose of Empagliflozin can alter the outcomes.

It was apparent that the differences regarding mortality became visible in the early duration, within 6 weeks after randomization. However, the time correlated with the period during which the differences between A1c levels between groups were maximum as well. Moreover, as the gaps for A1c between groups narrowed, both all cause and cardiovascular mortality curves flattened as well. Therefore, better glycemic control may have 
contributed to the differences in all cause mortality in the earlier period in the trial either directly or via consequential improvement in both lipid profiles and other cardiovascular surrogate markers. Similarly, the improvement in outcomes, if it's real, may also be attributed to the lower blood pressure achieved in Empagliflozin groups. These findings may be consistent with the data documented in UKPDS and other studies which showed lowering of all cause mortality and other cardiovascular outcomes with lowering of $\mathrm{HbAlc}$ and blood pressure in both the short and the long terms [2]-[5]. However, even in these studies, there was no difference in outcomes after such a short duration of intervention described in this study despite improvement in glycemic control. In fact, the duration at which the difference in cardiovascular outcomes between intensive intervention policy and the conventional treatment policy was noted was markedly longer in UKPDS. Therefore, this finding of improved outcomes after such a short duration of treatment with Empagliflozin is very difficult to fathom or accept and hence is "too good to be true". It is likely that the differences in outcomes between Empagliflozin groups on one hand and subjects treated with placebo group on the other may be secondary to the differences in the demographics of the subjects in these groups. It is likely that the subjects in placebo group may be older, with longer duration of diabetes, with more comorbidities including both micro and macro vascular complications and especially history of previous cardiovascular events. The provision of this data is extremely important and distinctly crucial especially in the light of unacceptable high rate of dropouts which primarily occurred in the early period of the duration of the trial. The other reason for provision of this data is the reaching of the plateau for the outcomes between all groups after this early period followed by the persistence of this plateau over the rest of the duration of the study. Moreover, the difference in outcomes could have been attributed to Empagliflozin only if the glycemic control and the blood pressure readings were comparable throughout the duration of the study period thus eliminating the impact of these other variable factors. However, neither the comparable glycemic control nor the non significant blood pressure readings were attained and maintained in the placebo group in comparison to subjects administered Empagliflozin. This finding is a distinctly surprising fact since subjects treated with placebo were also administered several other anti hyperglycemic drugs including insulin as well as many other antihypertensive agents. Therefore, It is likely that several study sites did not manage their subjects with "treat to target" strategy for both the glycemic control as well as hypertension. Alternatively, unacceptable reoccurrence of hypoglycemia or untoward side effects of antihypertensive drugs in subjects treated with placebo may have contributed to the lack of attainment and maintenance of desirable or at the least, non inferior glycemic control and blood pressure readings in comparison to Empagliflozin groups. And the role of hypoglycaemia in the induction of sudden death probably due to onset of arrhythmia cannot be overstated as is recently implicated in several studies and hence cannot be excluded [6]-[12]. The role of dysrhythmias in the deaths cannot be overlooked either since the deaths could not be attributed to myocardial infarction or unstable angina since differences between groups for these outcomes were not significantly different. In fact, the incidence of strokes was significantly higher in subjects treated with Empagliflozin when compared with the placebo group. Thus, once again, it is difficult to understand the differences between cardiovascular outcomes between the Empgliflozin group and the subjects administered placebo. Alternatively, multinational and multi regional nature of the study though ambitious, carries a burden of including subjects with variable backgrounds including race, ethnicity, genetic characteristics, dietary patterns and habits as well as the other diverse factors such as age at onset, differences in degree of obesity, rapid progression of the disorder and a major pathophysiologic mechanism of the disorder namely decline in insulin secretion in nonobese in contrast to markedly rising insulin resistance in obese population [13] [14]. Therefore, it would have been prudent and appropriate to provide the aforementioned variable factors as well as plasma glucose, A1c, lipid profiles and concentrations of other cardiovascular surrogate markers on admission prior to demise of these subjects. Moreover, the deaths and other adverse events may have been caused by markedly altered serum concentrations of electrolytes, $\mathrm{Mg}, \mathrm{Ca}, \mathrm{P}$ and acid base status since these abnormalities are established to be induced by additional drugs used in the placebo group to improve both the glycemic control and attain desirable blood pressure goals and these laboratory abnormalities are also well documented to promote cardiac conduction disruptions as well as dysrhythmias. Therefore, the documentation of these serum chemistries prior to or at the time of demise of the subjects may have provided a greater clarity regarding the exact cause of death especially in the subjects administered placebo. It is likely that more subjects in placebo group may have experienced these serum abnormalities as compared to subjects administered Empagliflozin because of the more frequent administration of the antihypertensive agents such as diuretics as well as drugs inhibiting RAAS system as well as antihyperglycemic drugs including insulin. The differences between groups are further exaggerated by expressing the rates of deaths/1000 patient years rather than as events 
per number of subjects in individual groups. Furthermore, it may be inappropriate to include deaths with unknown cause as cardiovascular deaths. Similar issues raised in this commentary are voiced recently in another publication as well [15]. Therefore, the conclusion of the study is "too good to be true" as described in this commentary including several flaws in the design of the study protocol, inclusion of subjects with extremely variable characteristics in multiple sites in several regions of the world with treatment decisions left to the discretion of individual investigators with variable philosophies regarding clinical practices and desirable goals of management. Finally, several gaps in documentation of important data parameters including occurrence of many disorders contributing to both the increased morbidity and mortality e.g. ketoacidosis, severe urinary sepsis, dehydration, fractures etc. in subjects treated with Empagliflozin despite being announced as concerns and alerts previously and reissued recently by FDA [16] [17] further adds to the skepticism expressed in this commentary regarding the conclusions of the authors.

\section{References}

[1] Zinman, B., Wanner, C., Lachin, J.M., Fitchett, D., Bluhmki, E., Hantel, S., Mattheus, M., Devins, T., Johansen, O.E., Woerle, H.J., Broedl, U.C. and Inzucchi, S.E., EMPA-REG Outcome Investigators (2015) Empagliflozin, Cardiovascular Outcomes, and Mortality in Type 2 Diabetes. The New England Journal of Medicine, 373, 2117-2128.

[2] UK Prospective Diabetes Study (UKPDS) Group (1998) Intensive Blood-Glucose Control with Sulphonylureas or Insulin Compared with Conventional Treatment and Risk of Complications in Patients with Type 2 Diabetes (UKPDS 33). The Lancet, 352, 837-853. http://dx.doi.org/10.1016/S0140-6736(98)07019-6

[3] UK Prospective Diabetes Study (UKPDS) Group (1998) Effect of Intensive Blood-Glucose Control with Metformin on Complications in Overweight Patients with Type 2 Diabetes (UKPDS 34). The Lancet, 352, 854-865. http://dx.doi.org/10.1016/S0140-6736(98)07037-8

[4] Kabadi, U.M. (2002) United Kingdom Prospective Diabetes Study: A Different Perspective. Endocrine Practice, 8, 6164. http://dx.doi.org/10.4158/EP.8.1.61

[5] Holman, R.R., Paul, S.K., Bethel, M.A., Matthews, D.R. and Neil, H.A. (2008) 10-Year Follow-Up of Intensive Glucose Control in Type 2 Diabetes. The New England Journal of Medicine, 359, 1577-1589. http://dx.doi.org/10.1056/NEJMoa0806470

[6] Seaquist, E.R., Miller, M.E., Bonds, D.E., Feinglos, M., Goff Jr., D.C., Peterson, K. and Senior, P., ACCORD Investigators (2012) The Impact of Frequent and Unrecognized Hypoglycemia on Mortality in the ACCORD Study. Diabetes Care, 35, 409-414. http://dx.doi.org/10.2337/dc11-0996

[7] Hanefeld, M., Duetting, E. and Bramlage, P. (2013) Cardiac Implications of Hypoglycaemia in Patients with Diabetes: A Systematic Review. Cardiovascular Diabetology, 21, 135.

[8] Tsujimoto, T., Yamamoto-Honda, R., Kajio, H., Kishimoto, M., Noto, H., Hachiya, R., Kimura, A., Kakei, M. and Noda, M. (2014) Vital Signs, QT Prolongation, and Newly Diagnosed Cardiovascular Disease during Severe Hypoglycemia in Type 1 and Type 2 Diabetic Patients. Diabetes Care, 37, 217-221. http://dx.doi.org/10.2337/dc13-0701

[9] Chow, E., Bernjak, A., Williams, S., Fawdry, R.A., Hibbert, S., Freeman, J., Sheridan, P.J. and Heller, S.R. (2014) Risk of Cardiac Arrhythmias during Hypoglycemia in Patients with Type 2 Diabetes and Cardiovascular Risk. Diabetes, 63, 1738-1747. http://dx.doi.org/10.2337/db13-0468

[10] Khunti, K., Davies, M., Majeed, A., Thorsted, B.L., Wolden, M.L. and Paul, S.K. (2015) Hypoglycemia and Risk of Cardiovascular Disease and All-Cause Mortality in Insulin-Treated People with Type 1 and Type 2 Diabetes: A Cohort Study. Diabetes Care, 38, 316-322. http://dx.doi.org/10.2337/dc14-0920

[11] Pistrosch, F. and Hanefeld, M. (2015) Hypoglycemia and Cardiovascular Disease: Lessons from Outcome Studies. Current Diabetes Reports, 15, 117. http://dx.doi.org/10.1007/s11892-015-0678-2

[12] Elwen, F.R., Huskinson, A., Clapham, L., Bottomley, M.J., Heller, S.R., James, C., Abbas, A., Baxter, P. and Ajjan, R.A. (2015) An Observational Study of Patient Characteristics and Mortality Following Hypoglycemia in the Community. BMJ Open Diabetes Research \& Care, 3, Article ID: e000094.

[13] India Diabetes Management Algorithm Proposal Group (2016) A Proposed India-Specific Algorithm for Management of Type 2 Diabetes. Diabetes Technology and Therapeutics, 18, 1-5.

[14] Kabadi, U.M. (2015) Major Pathophysiologic Factor in Prediabetes and Type 2 Diabetes Mellitus: Decreased Insulin Secretion in Lean and Insulin Resistance in Obese subjects. Abstract No. 2016, Endocrine Society Annual Meeting.

[15] Naci, H., Basu, S. and Yudkin, J.S. (2015) Preventing Cardiovascular Events with Empagliflozin: At What Cost? The Lancet Diabetes \& Endocrinology, 3, 931. http://dx.doi.org/10.1016/S2213-8587(15)00439-8

[16] US Food and Drug Administration (2015) Drug Safety Communication: FDA Warns That SGLT2 Inhibitors for Di- 
abetes May Result a Serious Condition of Too Much Acid in Blood. 15 May 2015. http://www.fda.gov/downloads/Drugs/DrugSafety/UCM446954.pdf

[17] FDA Drug Safety Communication (2015) FDA Revises Labels of SGLT2 Inhibitors for Diabetes to Include Warnings about Too Much Acid in the Blood and Serious Urinary Tract Infections. Posted 12 April 2015. 